\title{
People over forty feel $20 \%$ younger than their age: Subjective age across the lifespan
}

\author{
DAVID C. RUBIN \\ Duke University, Durham, North Carolina \\ and \\ DORTHE BERNTSEN \\ University of Aarhus, Aarhus, Denmark
}

\begin{abstract}
Subjective age - the age people think of themselves as being-is measured in a representative Danish sample of 1,470 adults between 20 and 97 years of age through personal, in-home interviews. On the average, adults younger than 25 have older subjective ages, and those older than 25 have younger subjective ages, favoring a lifespan-developmental view over an age-denial view of subjective age. When the discrepancy between subjective and chronological age is calculated as a proportion of chronological age, no increase is seen after age 40; older respondents feel $20 \%$ younger than their actual age. Demographic variables (gender, income, and education) account for very little variance in subjective age.
\end{abstract}

Research has shown that most adults think of themselves as younger than their actual chronological age (e.g., Kastenbaum, Derbin, Sabatini, \& Artt, 1972; Öberg \& Tornstam, 2001). Cross-sectional studies of self-perceived or subjective age across the lifespan show an increasing discrepancy between subjective age and actual age as people grow older (e.g., Goldsmith \& Heiens, 1992; Kastenbaum et al., 1972; Öberg \& Tornstam, 2001). This discrepancy is generally regarded as a denial of aging that is most pronounced in old age (e.g., Barak, Mathur, Lee, \& Zhang, 2001; Peters, 1971; Ward, 1977). Montepare and Lachman (1989) have summarized this view: "Theorists in the aging field have suggested that the tendency of aging adults to maintain younger subjective age identities is a form of defensive denial by which they can dissociate themselves from the stigma attached to growing old" (p. 73). Following this age-denial view, a youthful subjective age is an indicator of successful aging (e.g., Uotinen, Suutama, \& Ruoppila, 2003), which is consistent with studies showing that subjective age is a better predictor of psychological and physical functioning in old age than is chronological age (e.g., Barak \& Stern, 1986; Markides \& Boldt, 1983; Peters, 1971; but see Knoll, Rieckmann, \& Scholz, 2004). Under this age-denial view, there is no reason to suspect a discrepancy between subjective and chronological age in childhood and early adulthood, but the discrepancy should accelerate with older ages.

This work was supported by a grant from the Danish Research Council for the Humanities and by National Institutes of Health Grant R01 MH066079. We thank Professors Barak and Wixted for their comments. Correspondence concerning this article should be addressed to D. C. Rubin, Psychological and Brain Sciences, Duke University, Box 90086, Durham, NC 27708-0086 (e-mail: david.rubin@duke.edu).
An alternative view incorporates changes over the entire lifespan. Under this view and using purposely vague terms that we will clarify, people younger than "a certain age" want to be "a bit" older and people older than that age want to be "a bit" younger. Thus, under what we term a lifespan-developmental view, a discrepancy between chronological age and subjective age is not primarily an aging phenomenon (e.g., Galambos, Kolaric, Sears, \& Maggs, 1999; Montepare, 1996). To make sense of this, we have to specify the terms "a certain age" and "a bit." A review of the subjective age literature converges on the observation that "a certain age," which we term an attractor age, is somewhere in early adulthood (Montepare, 1996), a period that has the highest density of normative transition events (Berntsen \& Rubin, 2004; Neugarten, Moore, \& Lowe, 1965; Rubin \& Berntsen, 2003), is important to identity and its effects on cognition (Conway \& Pleydell-Pearce, 2000; Sehulster, 1996), and has the most available autobiographical memories (Rubin, Rahhal, \& Poon, 1998). Here we take an empirical approach and let the data determine the exact attractor age. The term "a bit" might have two meanings: a discrepancy or a proportional discrepancy. For example, a 75-year-old person may feel "a bit" younger if he or she feels $20 \%$, or 15 years, younger. A 30-year-old person may feel "a bit" younger if he or she feels $20 \%$, or 6 years, younger. In contrast, a 30 -year-old person who feels the same 15 years younger as did the 75-year-old person appears to feel more than "a bit" younger. Thus, for an entire lifespan, a proportional view may be more reasonable. Here, we turn the lifespan-developmental view into a quantitative statement by assuming that people of all ages will tend to have a subjective age that is closer to an empirically determined attractor age in early adulthood and that the further they are from the attractor age the greater their proportional 
discrepancy will be up to a maximum amount, which we will also determine empirically.

Few studies have systematically examined how subjective age varies with the age of the participant over the adult lifespan. Öberg and Tornstam (2001) found an increasing discrepancy between subjective and chronological age measured in years with increasing chronological age in a study with 1,250 Swedes. However, no detailed analyses were conducted on this distribution. Goldsmith and Heiens (1992) showed that with increasing chronological age, an increasing number of participants in the U.S. judged their subjective age to be younger than their actual age. However, this study involved few participants at the oldest ages and did not measure subjective age in years. Other studies in which smaller convenience samples were used have presented similar findings (e.g., Barnes-Farrell \& Piotrowski, 1989; Kaliterna, Larsen, \& Brkljacic, 2002; Kastenbaum et al., 1972; Montepare \& Lachman, 1989). In the present study, we provide detailed quantitative descriptions and systematic analyses of how subjective age varies with the age of the participant over the adult lifespan by using a representative sample of a large number of adults.

Subjective age has been operationalized differently across different studies (see Barak \& Stern, 1986, for a review). In most studies, participants have described their subjective age in terms of decades - that is, "I feel as though I am in my twenties." Some studies have used a compound score for subjective age by averaging people's answers to questions addressing how old they look, feel, and appear to be in terms of their interests and activities, whereas other studies have treated such measures separately. In some studies, only comparative age has been assessed; that is, participants are asked to assess whether they feel older than, younger than, or the same as their current age. Other studies have measured felt age by asking participants to estimate in years how old they feel inside. This measure is continuous and allows mathematically more sophisticated analyses to be conducted on the relation between subjective age and chronological age. We asked our respondents both their comparative age and their felt age in order to allow an analysis of both measures. From the comparative question, we found the attractor age, at which there are as many people feeling younger as older. From the felt-age question, we calculated three measures: felt age (FA), the discrepancy between felt age and chronological age (FA - CA), and proportional discrepancy, which is the discrepancy divided by age, or (FA - CA)/CA. The last measure is a variant of what Barak and Rahtz (1999), working within the age-denial view, called perceived youth, but proportional discrepancy is more theoretically neutral and applicable to younger ages.

Because past research on individual differences related to subjective age was characterized by conflicting findings and because we had a large, fairly representative sample of the Danish population, we also investigated the individualdifference measures of gender, income, education, and social economic status to try to help clarify this situation. For instance, some studies have shown an inverse relation between subjective age and education and income (see Barak \& Stern, 1986, for a review), whereas other studies have been unable to replicate these findings (Henderson, Goldsmith, \& Flynn, 1995). Some studies have shown that women perceive themselves as younger more often than men do (e.g., Montepare \& Lachman, 1989), some have shown no gender differences (e.g., Barak et al., 2001; Henderson et al., 1995), and some have shown that men perceive themselves as younger more often than women do (e.g., Cooper, Thomas, \& Stevens, 1981).

Thus, we ask three different kinds of questions about subjective age. First, what is the distribution of subjective age over the lifespan, and does it better support the age-denial or the lifespan-developmental view? The agedenial view predicts no discrepancies in early adulthood and accelerated discrepancies in old age. In contrast, the lifespan-developmental view predicts that discrepancies should be in the direction of an attractor age no matter what the age of the participant. Second, what is the relation between the comparative age and felt age questions used in the subjective age literature? Third, are individual differences in subjective age related to individual differences in gender, social economic status, income, and education?

\section{METHOD}

A representative sample of 1,485 Danes (20 to 97 years of age) participated as part of an omnibus survey by Gallup Public, Denmark, of which 1,470 provided answers to the questions asked here. In each household, 1 or 2 respondents were randomly selected via a combined criterion based on number of household members above age 16 and their birthdays. Response rates for the entire omnibus survey were $58 \%$. Questions about when emotional events occurred were also included and are reported in Berntsen and Rubin (2004).

The data were collected by 78 interviewers via face-to-face interviews in the respondents' homes. The interviewers recorded responses on a laptop computer. To ensure understanding, each question and the response options for each question were printed on a demonstration card, which was shown to the respondent while the question was being asked. Or if the respondent preferred, he or she could read the questions and response options directly from the computer screen. In addition to general questions about age, gender, education, income, and occupation, which were part of the omnibus survey, two questions are analyzed here. The first addressed comparative age: "This question is about how old you feel inside. Some people feel either older or younger inside than their actual age. Other people do not feel older or younger than their actual age. How old do you, yourself, feel inside?" The question had three possible answers: "I feel younger," "I feel older," and "I feel neither older or younger." If the response was either I feel younger or older, the second question addressing felt age was asked: "You feel that you are a different age inside than the one from your birth certificate. What age do you feel you are inside?" The question had a response of the form "Inside I feel around years old."

\section{RESULTS}

For the analyses shown in Figures 1, 2, and 3, the respondents were grouped into bins of 5 years up to age 64 and into bins of about 10 years for older ages, for which there were fewer respondents. The number of respondents in each bin, along with their mean age and demographic variables, are given in Table 1 . As these variables were de- 
Table 1

Respondents

\begin{tabular}{ccccccc}
\hline $\begin{array}{c}\text { Age } \\
\text { Range }\end{array}$ & \multicolumn{1}{c}{$n$} & $\begin{array}{c}\text { Mean } \\
\text { Age }\end{array}$ & $\begin{array}{c}\text { Percent } \\
\text { Females }\end{array}$ & Education & Income & $\begin{array}{c}\text { Social } \\
\text { Group }\end{array}$ \\
\hline $20-24$ & 90 & 22.21 & 51 & 4.12 & 3.19 & 2.39 \\
$25-29$ & 134 & 27.03 & 51 & 5.08 & 4.05 & 1.85 \\
$30-34$ & 142 & 32.12 & 42 & 5.19 & 4.94 & 1.61 \\
$35-39$ & 149 & 36.86 & 52 & 5.13 & 5.28 & 1.63 \\
$40-44$ & 139 & 41.99 & 56 & 5.38 & 5.20 & 1.63 \\
$45-49$ & 118 & 47.08 & 56 & 5.14 & 5.12 & 1.59 \\
$50-54$ & 136 & 52.04 & 46 & 5.35 & 5.23 & 1.59 \\
$55-59$ & 143 & 57.03 & 45 & 4.62 & 4.74 & 1.71 \\
$60-64$ & 110 & 61.97 & 51 & 4.08 & 3.58 & 2.09 \\
$65-74$ & 163 & 69.13 & 60 & 3.71 & 2.78 & 2.51 \\
$75-84$ & 100 & 79.30 & 58 & 3.58 & 1.98 & 2.55 \\
$85-97$ & 46 & 88.11 & 87 & 2.17 & 1.67 & 2.78 \\
\hline
\end{tabular}

fined by Gallup, education ranged from 1 ( 7 years or less of schooling) to 5 (basic vocational training) to 7 (more than 3 years of higher education); income ranged from 1 (less than 100,000 kroner/year) to $5(400,000-500,000$ kroner/year) to 8 (more than 700,000 kroner/year); and social group was a 3-point scale, with 1 being the highest class.

The proportion of respondents who replied that their subjective age was younger than, the same as, or older than their current age is shown in Figure 1. Consistent with the lifespan-developmental view, with increasing age up to age 40 , the proportion of older subjective age responses drops to about .02. With increasing age up to age 40 , the proportion of younger subjective age responses increases to about .70. The proportion of respondents who said that their subjective age was equal to their chronological age was highest for the youngest respondents and dropped to about .30. The crossover where the same proportion of people feel younger and older is at about 25 years old, our empirical determination of the attractor age.

Figure 2 shows the distribution of felt age as a function of the respondents' chronological age. A theoretical line of felt age equal to chronological age is also shown. Consistent with the lifespan-developmental view and Figure 1, up to age 25 , felt age is higher than chronological age, after which point felt age become lower than chronological age. Measured in years, it appears that the discrepancy between felt and chronological age increases as our respondents become older, consistent with previous studies (e.g., Öberg \& Tornstam, 2001), which has led to the discrepancy being viewed as most pronounced in older adults (e.g., Barak et al., 2001; Goldsmith \& Heiens, 1992; Kaliterna et al., 2002). However, as is illustrated by Figure 3, when proportional discrepancy was plotted, no increase was seen after age 40; after age 40 , respondents across all age groups reported that they felt roughly $20 \%$ younger than their actual age. If the $30 \%$ of the respondents who reported no discrepancy were included the value would be about $14 \%$.

Our data suggest that choosing to say one feels a different age and estimating that age are different psychological processes. As is shown in Figure 1, about 30\% of the respondents answered that they felt no age discrepancy. If these respondents were simply estimating their felt age and determining that it equaled their chronological age, we would expect a substantial proportion of felt age responses near to people's chronological age. That is, we would expect a normal distribution centered around the respondents' chronological age for a good percentage of our respondents. But this is not what we observe. If only the 955 respondents over age 39 are included, $70 \%$ of the respondents said that they felt younger, $27 \%$ said that they felt exactly the same age, but only $2 \%$ said that they felt older. If the respondents were making comparative age judgments based on felt age, one might expect greater than $2 \%$ to have responses slightly older than their age, given that $27 \%$ had zero discrepancies. Among the respondents over age 39, the 694 who felt younger or older had a mean discrepancy of -12.32 years $(S D=10.52)$, and so the mean of their distribution could not be a zero discrepancy $[t(693)=30.83, p<.0001]$. Yet $27 \%$ of this age group reported a zero discrepancy when they reported that they felt their chronological age.

Individual-difference analyses were performed to investigate the effects of gender and social economic status measures. To see whether respondents who responded that

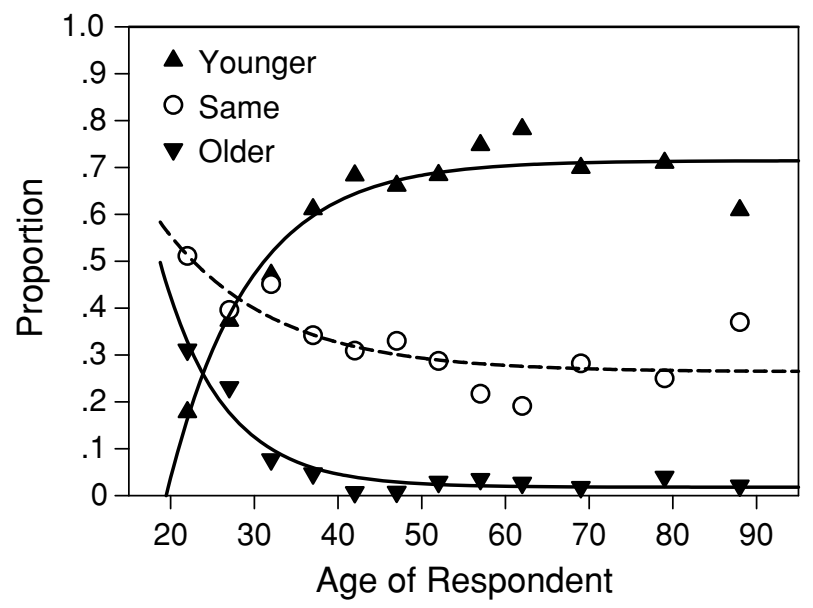

Figure 1. Comparative age: The proportion of respondents who reported that they felt as if they were younger than, the same as, or older than their actual age. 


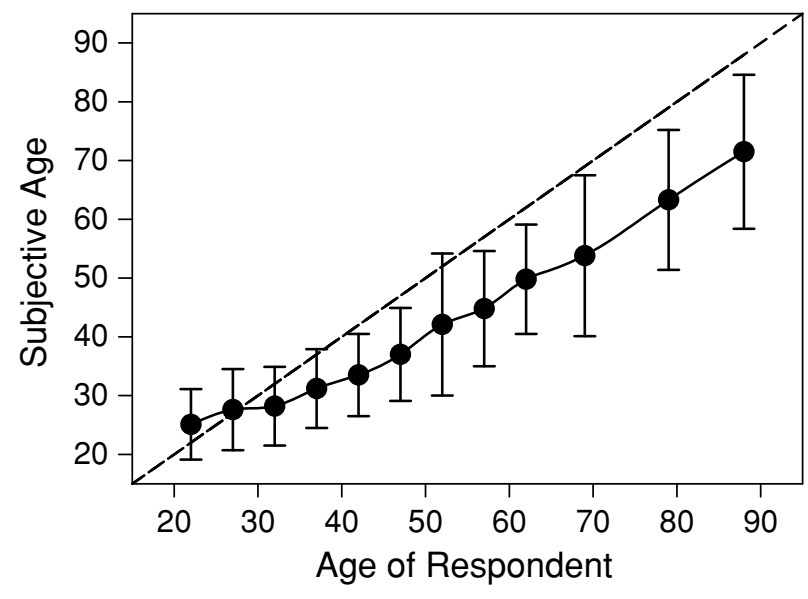

Figure 2. Felt age for respondents who reported feeling younger or older than their actual age. Error bars are standard deviations.

they felt an age other than their chronological age were different from those who did not, we used comparative age judgments to divide our respondents into those who reported subjective ages equal to their actual age $(n=475)$ and those who reported either younger or older ages $(n=$ 995). ANOVAs performed using this split with the dependent variables of education and income were not significant $[F(1,1463)=0.00, p=.97$, and $F(1,1307)=1.95$, $p=.16$, respectively]. Chi-square analyses for the dichotomous gender and the three-level social group variables were also not significant $\left[\chi^{2}(1, N=1,470)=0.25, p=\right.$ .62 , and $\chi^{2}(2, N=1,369)=3.82, p=.15$, respectively]. There was an effect of chronological age $[F(1,1468)=$ 27.33, $p<.0001$ ], which, as is shown in Figure 1, was due to the fact that the proportion of people who reported subjective ages equal to their chronological age decreased with age. Having found no individual differences other than age in whether the respondents did or did not report a subjective age with a discrepancy, we next examined responses of those respondents who did.

We performed regression analyses with felt age, discrepancy, and proportional discrepancy as dependent variables and age, gender, and social economic status as independent variables. In separate analyses, we replaced social economic status with two of the scales that contributed to it: years of education and income. In order to look for any possible effects, we performed 12 regression equations produced by crossing our three dependent measures (felt age, discrepancy, and proportional discrepancy) with two samples (including or excluding respondents with subjective age equal to chronological age) with two sets of individual-difference variables (either social group or the income and education which are included in it). Because of the nonlinear trend in Figure 2, an age-squared predictor was added. There were effects of chronological age and age squared, as is shown in Figures 2 and 3. But even with the large number of respondents and uncorrected multiple comparisons, gender, education, and social economic status did not enter into any of the regressions at the .05 level. Income did enter into all six regression equations in which it was a predictor, adding, at most, .006 to the $r^{2}$ of the regression equation.

Thus, with the exception of the effects of chronological age, the demographic measures had, at most, a minor role in accounting for comparative age and all three measures based on felt age, a finding that is consistent with some previous studies (e.g., Barak et al., 2001; Henderson et al., 1995).

\section{DISCUSSION}

The present study has five main findings. First, the lifespan-developmental view provides a better account of the data than does the dominant age-denial view. The discrepancy between subjective age and chronological age does not simply increase across the lifespan, but rather,

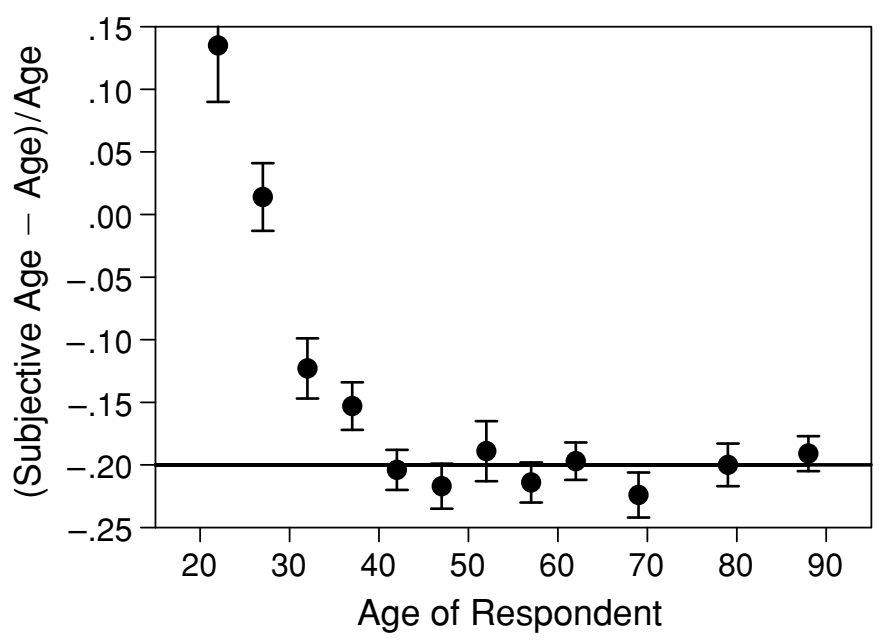

Figure 3. Age discrepancy as a proportion of age. Error bars are standard errors. 
younger respondents feel older and older respondents feel younger. Second, the attractor age or crossover point between feeling older and feeling younger is 25 . Third, when the discrepancy between felt age and chronological age is normalized by dividing it by chronological age, no increase is seen after age 40 . After this age, all age groups feel an average $20 \%$ younger than their actual age.

Fourth, reporting that you feel younger or older than your actual age and estimating that difference appear to be different kinds of judgments. Too many respondents reported that they felt their own age, given the size and consistency of the discrepancies reported by those respondents who felt a different age. Thus, it is problematic to generalize across studies that use measures of comparative age and felt age or to report on felt age without also reporting separately on those respondents who had nonzero discrepancies, because these measures appear to probe different assessments.

Fifth, we investigated the effects of gender, social economic status, education, and income on comparative age and three measures of subjective age and found no effects for gender, social economic status, and education and only minimal effects of income. This is despite a large sample size and performing multiple tests without correcting the $p$ level for multiple comparisons. Although not directly related to the other theoretical points we tried to make here, the lack of gender differences in any of our measures is of note.

Although the dominant view in the study of subjective age has been age denial, we believe that a lifespandevelopmental view is needed. Such a view better describes the data, which should facilitate theoretical advancement, as well as serving as a practical description in applied areas, such as marketing. In contrast to age denial, we found that people of all ages tend to have a subjective age that is closer to an attractor age of 25 years and that the further they are from this attractor age the greater their proportional discrepancy is up to a maximum of $20 \%$. Future research may revise the estimates of an attractor age of 25 years and the maximum proportional discrepancy of $20 \%$, and it is likely that these two parameters will vary with the culture of the populations tested. But we expect that the basic findings shown in Figures 1 and 3 will hold.

\section{REFERENCES}

Barak, B., Mathur, A., Lee, K., \& Zhang, Y. (2001). Perceptions of age-identity: A cross-cultural inner-age exploration. Psychology \& Marketing, 18, 1003-1029.

BARAK, B., \& RAHTZ, D. (1999). Perceived youth: Appraisal and characterization. International Journal of Aging \& Human Development, 49, 231-257.

BaraK, B., \& Stern, B. (1986). Subjective age correlates: A research note. Gerontologist, 26, 571-578.
Barnes-Farrell, J. L., \& Piotrowski, M. J. (1989). Workers' perceptions of discrepancies between chronological age and personal age: You're only as old as you feel. Psychology \& Aging, 4, 376-377.

Berntsen, D., \& Rubin, D. C. (2004). Cultural life scripts structure recall from autobiographical memory. Memory \& Cognition, 32, 427442.

Conway, M. A., \& Pleydell-Pearce, C. W. (2000). The construction of autobiographical memories in the self-memory system. Psychological Review, 107, 261-268.

Cooper, P. E., Thomas, L. E., \& Stevens, S. J. (1981). Subjective time experience in an intergenerational sample. International Journal of Aging \& Human Development, 13, 183-193.

Galambos, N. L., Kolaric, G. C., Sears, H. A., \& Maggs, J. L. (1999). Adolescents' subjective age: An indicator of perceived maturity. Journal of Research on Adolescence, 9, 309-337.

Goldsmith, R. E., \& Heiens, R. A. (1992). Subjective age: A test of five hypotheses. Gerontologist, 32, 312-317.

Henderson, K. V., Goldsmith, R. E., \& Flynn, L. R. (1995). Demographic characteristics of subjective age. Journal of Social Psychology, 135, 447-457.

Kaliterna, L., Larsen, Z. P., \& Brkljacic, T. (2002). Chronological and subjective age in relation to work demands: Survey of Croatian workers. Experimental Aging Research, 28, 39-49.

Kastenbaum, R., Derbin, V., Sabatini, P., \& Artt, S. (1972). "The ages of me": Toward personal and interpersonal definitions of functional aging. International Journal of Aging \& Human Development, 3, 197-211.

KnOll, N., Rieckmann, N., \& Scholz, U. (2004). Predictors of subjective age before and after cataract surgery: Conscientiousness makes a difference. Psychology \& Aging, 19, 676-688.

Markides, K. S., \& BoldT, J. S. (1983). Change in subjective age among the elderly: A longitudinal analysis. Gerontologist, 23, 422-427.

Montepare, J. M. (1996). Variations in adults' subjective ages in relation to birthday nearness, age, age awareness, and attitudes toward aging. Journal of Adult Development, 3, 193-203.

Montepare, J. M., \& Lachman, M. E. (1989). "You are only as old as you feel": Self-perceptions of age, fears of aging, and life satisfaction from adolescence to old age. Psychology \& Aging, 4, 73-78.

Neugarten, B. L., Moore, J. W., \& Lowe, J. C. (1965). Age norms, age constraints, and adult socialization. American Journal of Sociology, 70, 710-717.

ÖBerg, P., \& Tornstam, L. (2001). Youthfulness and fitness-identity ideals for all ages? Journal of Aging \& Identity, 6, 15-29.

Peters, G. R. (1971). Self-conceptions of the aged, age-identification, and aging. Gerontologist, 11, 69-73.

Rubin, D. C., \& Berntsen, D. (2003). Life scripts help to maintain autobiographical memories of highly positive, but not highly negative, events. Memory \& Cognition, 31, 1-14.

Rubin, D. C., Rahhal, T. A., \& Poon, L. W. (1998). Things learned in early adulthood are remembered best. Memory \& Cognition, 26, 3-19.

SeHUlster, J. R. (1996). In my era: Evidence for the perception of a special period of the past. Memory, 4, 145-158.

Uotinen, V., Suutama, T., \& Ruoppila, I. (2003). Age identification in the framework of successful aging: A study of older Finnish people. International Journal of Aging \& Human Development, 56, 173-195.

WARD, R. A. (1977). The impact of subjective age and stigma in older persons. Journal of Gerontology, 32, 227-232.

(Manuscript received October 18, 2005; revision accepted for publication January 23, 2006.) 